\title{
Trajectory Extraction and Deep Features for Classification of Liquid-Gas Flow under the Context of Forced Oscillation
}

\author{
Luong Phat Nguyen ${ }^{1}$, Julien Mille ${ }^{1,2}$, Dominique $\mathrm{Li}^{1}$, Donatello Conte ${ }^{1}$, Nicolas Ragot ${ }^{1}$ \\ ${ }^{1}$ Université de Tours, Tours, France \\ 2 INSA Centre Val de Loire, Blois, France \\ luongphat.nguyen@etu.univ-tours.fr, \\ julien.mille@insa-cvl.fr, \\ \{dominique.li, donatello.conte, nicolas.ragot $\} @$ univ-tours.fr
}

Keywords: Video classification, dynamic texture, deep learning, liquid-gas flow

\begin{abstract}
Computer vision and deep learning techniques are increasingly applied to analyze experimental processes in engineering domains. In this paper, we propose a new dataset of liquid-gas flow videos captured from a mechanical model simulating a cooling gallery of an automobile engine, through forced oscillations. The analysis of this dataset is of interest for fluid-mechanic field to validate the simulation environment. From computer vision point of view, it provides a new dynamic texture dataset with challenging tasks since liquid and gas keep changing constantly and the form of liquid-gas flow is closely related to the external environment. In particular predicting the rotation velocity of the engine corresponding to liquid-gas movements is a first step before precise analysis of flow patterns and of their trajectories. The paper also provides an experimental analysis showing that such rotation velocity can be hard to predict accurately. It could be achieved using deep learning approaches but not with state-of-the-art method dedicated to trajectory analysis. We show also that a preprocessing step with difference of Gaussian (DoG) over multiple scales as input of deep neural networks is mandatory to obtain satisfying results, up to $81.39 \%$ on the test set. This study opens an exploratory field for complex tasks on dynamic texture analysis such as trajectory analysis of heterogeneous masses.
\end{abstract}

\section{INTRODUCTION}

In fluid mechanical engineering, liquid-gas flow analysis plays an important role. For example, the movements of coolant has a huge impact on the cooling of engines. However, the identification of liquid-gas flow patterns is a challenging problem for two reasons. First, it is hard and costly to observe directly the fluids inside the cooling gallery of automobile engine pistons. Next, fluids contain a mixture of liquid and gas, forming bubbles and droplets. Moreover, liquid and gas appearance keeps changing constantly because of external heat and forces. Such changes are closely related to the external environment. To be able to observe and analyze flow-gas patterns and their trajectories under mechanical constraints, the University of Zhejian, China, has designed an experimental mechanical process to simulate what can happen inside a cooling gallery. The fluids movements, that should correspond to rotation speed of the engine, are obtained thanks to forced oscillations of the gallery. The final goals to achieve is to analyze fluids flow inside this experimental simulation environment to be able to validate that it is a good reproduction of what is happening inside engines and next to analyze fluids motions depending on nature of fluids, mechanical constraints, etc. in order to optimize cooling of engines.

In this study, we focus on a first challenge from computer vision point of view, which is to evaluate the correlation between observed patterns of liquidgas flow and the rotation velocities of automobile engines. This is a first step toward the validation of the simulation environment and before precise analysis of patterns and their trajectories. This challenge is closely related to dynamic texture analysis and trajectory analysis of heterogeneous masses.

Dynamic textures are visual cues that present characteristics in both spatial and temporal domains. They have rich content, and are thus spread in a highdimensional space. Extraction of spatio-temporal features and patterns may allow the characterization of observable movements. Then, these descriptions of dynamic textures can be used for applications such 
as video segmentation, classification or retrieval, but also to analyze mechanical cohesion as for a set of objects which form an heterogeneous mass, such as human crowds, swarms, school of fishes, flock of birds, or fluids.

The contributions of this work rely on three parts:

- We introduce a brand-new dataset based on twophased flow video captures, which is called Automobile Engine Rotation Velocity dataset. This one could be of interest for computer vision community since it allows several challenging tasks that are not often proposed in classical benchmarks (ie. analysis of flow patterns and their trajectories).

- From this dataset, we study two state-of-the-art video classification methods to evaluate the correlation between motor speeds and spatio-temporal information corresponding to the two-phase flow liquid movements in the dataset. The first method uses state-of-the-art handcrafted features, based on optical flow and dense trajectories, whereas the second one is based on ConvNets. This analysis is showing that trajectories, that can be seen as higher level representations interesting for human analysis, are either inadequate or need to be specifically adapted, which represent a new challenging task to be tackled. On the contrary, deep networks are more efficient but less explainable.

- To make this deep approach really efficient, we propose as a third contribution to improve them using a preprocessing method based on specularities inside videos. Consequently, an alternative challenging task brought by this study is how to provide precise analysis of flow patterns and their trajectories with deep features.

The organization of this paper is as follows. We deal with the related work on dynamic texture classification in videos in section 2 . We then introduce the new dataset on two-phase flow visualization in cooling gallery under forced oscillation in section 3. Section 4 presents the proposed methods used to classify the motor speed by using deep learning and optical flow based methods. Before we conclude our work in section 6, experimental setup and results are described out in section 5 .

\section{RELATED WORK}

Dynamic textures classification remains a challenging problem in computer vision. Many research contributions have been concentrated on the development of spatio-temporal features. There are two main categories: hand-crafted and deep learningbased methods. Early hand-crafted spatio-temporal feature-based methods depend on optical flow, such as (Nelson and Polana, 1992), (Renaud and Chetverikov, 2005), (Lu et al., 2007) and (Crivelli et al., 2013). However, dynamic textures are usually made by chaotic motions in several directions. So, optical flow-based methods, which lend themselves to the extraction of smooth motion fields, might not represent dynamic textures well.

Recently, Jansson et al. (Jansson and Lindeberg, 2018) propose a new family of video descriptors using a time-causal spatio-temporal scale-space framework for dynamic texture recognition. In the state of the art, joint histograms of spatio-temporal receptive field responses (first order and second order spatial and temporal derivatives) are calculated and then used as inputs of either a SVM or Nearest Neighbor classifier. This contribution shows that the time-causal and time-recursive receptive fields can achieve good results. However, this approach still does not obtain results as well as neural network-based methods (Jansson and Lindeberg, 2018) on classical benchmarks such as DynTex.

Since the breakthrough of AlexNet (Krizhevsky et al., 2017), a lot of deep learning approaches have been developed for dynamic texture classification. Some works are based on information that is purely spatial, like in (Qi et al., 2016), where 2D convolution filters are applied to each frame of a video. Such method neglects temporal regularity. A feature extraction on 3 orthogonal planes based on convolutional neural networks is used in (Andrearczyk and Whelan, 2018). Tran et al. (Tran et al., 2015) developed a 3D convolution neural network (C3D) for action recognition which achieves good results. Another deep learning approach in (Qiu et al., 2017) based on ResNet (P3D ResNet) uses spatio-temporal convolutional filters which are decomposed in spatial and temporal filters separately for learning spatiotemporal representation.

In this paper, we will investigate both approaches using classical methods such as the one of (Wang et al., 2013) for handcrafted features to characterize trajectories of particles, and a deep approach based on (Tran et al., 2018) which is an improvement of P3D nets. 


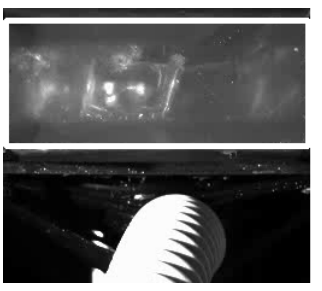

(a)

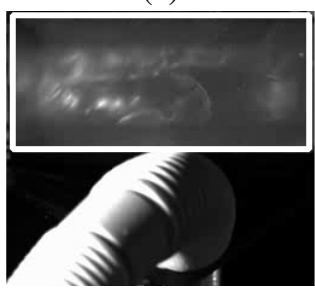

(c)

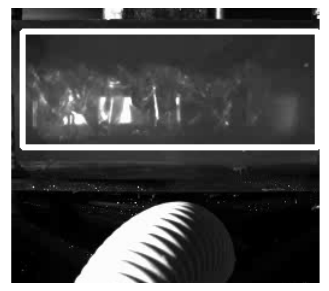

(b)

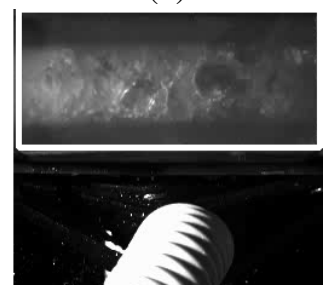

(d)
Figure 1: Liquid-gas flow visualization with the rotation velocities of (a) $200 \mathrm{rpm}$, (b) $300 \mathrm{rpm}$, (c) $400 \mathrm{rpm}$, and (d) $500 \mathrm{rpm}$.

\section{AUTOMOBILE ENGINE ROTATION VELOCITY DATA SET}

In this section, we introduce a new dataset of dynamic textures of fluid movements obtained thanks to an experimental engineering process that is simulating what is happening inside a cooling gallery of an automobile engine. The dataset contains 18 grayscale video clips (30 fps) and each clip has a duration of about 10 to 15 minutes. There are 9 classes, corresponding to the simulated rotation velocities of the engine. Each class is represented by two videos. In Figure 1, the movement of the liquid (glycerine) is shown for motor speeds of 200, 300, 400 and 500 rpm. For our experiments, the only interesting area in each video is the textures of the fluid inside the gallery. The tube and the surroundings should not be taken into account.

In order to extract the interesting areas in the videos, we used a traditional tracking method which is called 'Boosting' (Grabner et al., 2006). This algorithm is originally used for face tracking using a Haar cascade-based face detector. In the first frame of each video, we manually draw a bounding box around the interesting area. Then, this box is tracked and extracted in the rest of the video (see Figure 3).

Dataset structure. Our dataset contains 9 motor speed classes and each class contains 2 videos of 30 fps which last about 10 to 15 minutes. We downsample it in time by a factor of 3 , and divide each video into sequences of 30 consecutive frames without over-

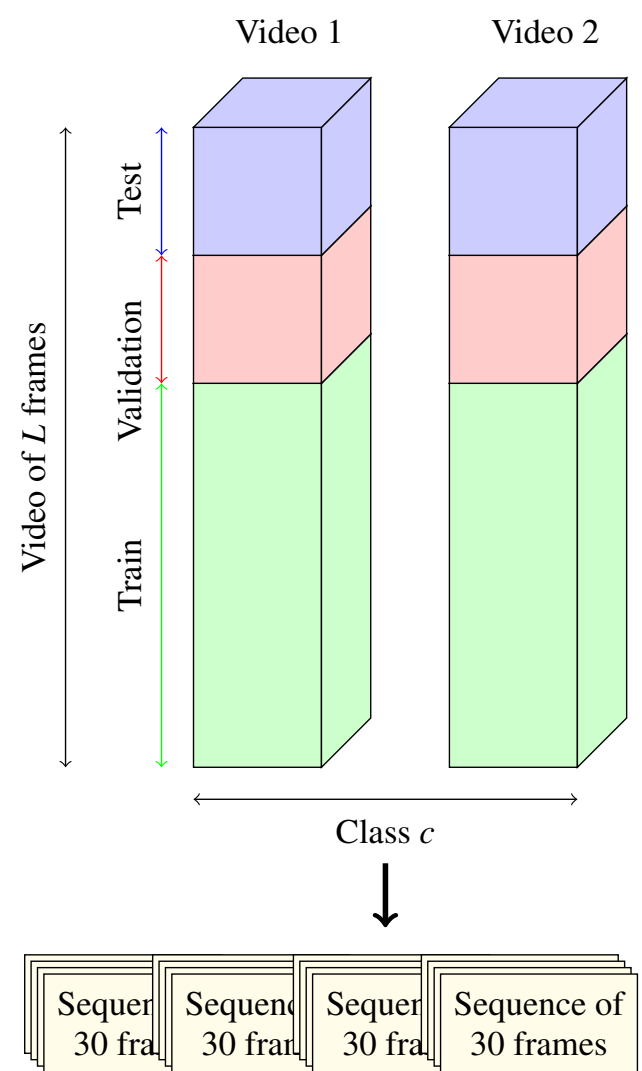

Figure 2: Dataset structure.

lapping. This gives us a dataset ${ }^{1}$ with around 6000 sequences of 30 consecutive frames. The dataset is split as follows: the first 60 percents of each video sequence are for training, the next $20 \%$ are for validation and the remaining ( $20 \%$ of the video) are for testing. Figure 2 shows the way how we divide our data. In the training process, we use the validation set to select the parameters giving the best accuracy. These parameters are then used for the test test.

\section{CLASSIFICATION OF COOLING GALLERY VIDEO SEQUENCES}

In this work, we compare two methods for our classification task:

- A method based on dense trajectories, optical flow and a SVM classifier.

\footnotetext{
${ }^{1}$ Due to the large size of the dataset (about $24 G b$ ), only the corresponding downsampled and cropped videos which contain only fluids textures are available at https://gitlab.com/lphatnguyen/ automobile-engine-rotation-velocity.
} 
- A deep network based on spatial and temporal convolutions.

\subsection{Dense Trajectories}

A first approach to classify two-phase flow videos is to track liquid particles and to model explicitly trajectories of interest points inside each video sequence of 30 frames. Indeed, modeling such trajectories could help engineers to analyze the fluid movements and its behaviour. This is the straight forward approach towards the final goal of the application domaine of this study. Among many traditional trajectory extraction algorithms, the method proposed by (Wang et al., 2013) has shown its effectiveness. In (Wang et al., 2013), keypoints are detected using a dense sampling method. These keypoints are then tracked in the following frames inside dense cubes along the trajectory. These cubes are subdivided by a spatio-temporal grid of size $n_{\tau} \times n_{\sigma} \times n_{\sigma}$. Features are extracted from these dense cubes to model each trajectory. These ones are clustered to generate Bag-of-Features, and then classification is performed using a SVM model.

The details of the approach is given here. First of all, points are sampled on a grid spaced by $\mathbf{w}$ pixels on a starting frame at multiple scales like in (Wang et al., 2013). However, all points on the grid cannot be tracked so these points are selected by using the criterion of (Jianbo Shi and Tomasi, 1994). This criterion helps to remove selected points if the eigenvalues of the auto-correlation matrix are too small. The threshold $T$ used is the same as in (Wang et al., 2013):

$$
T=0.001 \times \max _{(x, y) \in I}\left(\min \left(\lambda^{1}(x, y), \lambda^{2}(x, y)\right)\right)
$$

where $\lambda^{j}(x, y)$ is the $j^{t h}$ eigenvalue of the autocorrelation matrix around the pixel $(x, y)$. This method can be seen as an extension of the classical corner detection method.

Next, points are tracked to obtain trajectories, by means of a dense optical flow field, using Farnebäck algorithm (Farnebäck and Farneb, 2003). Furthermore, for a given tracked point, its new position is smoothed using a median filter of size $3 \times 3$. Median filter is used instead of bilinear interpolation (Wang et al., 2013) since it helps keeping sharp motion boundaries, especially in some locations such as borders. Here, one can notice that around 60 trajectories are extracted inside each video sequence (this may vary a lot depending on the sequence).

In order to describe a trajectory, represented by its dense cube, we use Histogram of Oriented Gradient (HOG) (Dalal and Triggs, 2005), Histogram of Optical Flow (HOF) (Laptev et al., 2008) and Motion Boundary Histogram (MBH) (Dalal et al., 2006) as motion and structure descriptors. HOG constructs the distribution of directions of oriented gradients, HOF calculates the histogram of directions of optical flows while MBH is actually the HOG applied to dense optical flow images.

Moreover, normalized displacement vector magnitudes are also used as dense trajectories features and are added to the final feature vector describing one trajectory.

After this step, one codebook (visual dictionary) is computed for each of the 5 types of trajectory feature vector (dense trajectories, $\mathrm{HOG}, \mathrm{HOF}, \mathrm{MBHx}$, MHBy). The construction of a codebook is done by clustering the trajectory feature vectors of all training sequences using K-means (Figure 4). The centroids found by K-means are the visual words used in the codebooks, and represent typical trajectories, for the considered feature type. We denote this visual dictionary by $\left\{w_{1}, \ldots w_{M}\right\}$, where $M$ is the chosen number of visual words, regardless of the considered feature type.

Then, for each video sequence of the training set, a bag-of-features (BoF) is created (Figure 5). Since there is an arbitrary number of trajectories within a sequence to be classified, the BoF approach allows to obtain a constant-size final feature vector for each sequence. $\mathrm{A} \mathrm{BoF}$ is a histogram counting the number of occurrences of each visual word in the codebook. The concatenation of the histograms (of the 5 features types) is used as the final feature vector for classification. This final feature vector is of size $5 M$. When constructing an histogram, each descriptor is assigned to the closest vocabulary word $w_{i}$ using Euclidean distance. The corresponding bin is increased.

For the classification step, we use the SVM algorithm with an RBF- $\chi^{2}$ kernel which is applied to BoF of sequences (Zhang et al., 2007). For two BoF vectors $\mathbf{a}, \mathbf{b} \in \mathbb{R}^{d}$, the $\chi^{2}$ kernel is

$$
K(\mathbf{a}, \mathbf{b})=\exp \left(-\gamma \sum_{i=1}^{d} \frac{\left(a_{i}-b_{i}\right)^{2}}{a_{i}+b_{i}}\right)
$$

In our case, the size of the BoF vectors is $d=5 M$.

\subsection{Deep Learning Methods}

\subsection{1 (2+1)D Convolution}

As mentioned in section 2, $\mathrm{R}(2+1) \mathrm{D}$ model has shown its efficiency in both accuracy and size. In (Tran et al., 2018), it is shown that a convolution in 3 dimensions can be decomposed into a 2D spatial convolution and a 1D temporal convolution as 2 successive steps. Let us consider a 3D convolution layer $i$ made of $N_{i}$ filters, each of size $N_{i-1} \times t \times d \times d$, where $t$ and $d$ represent 


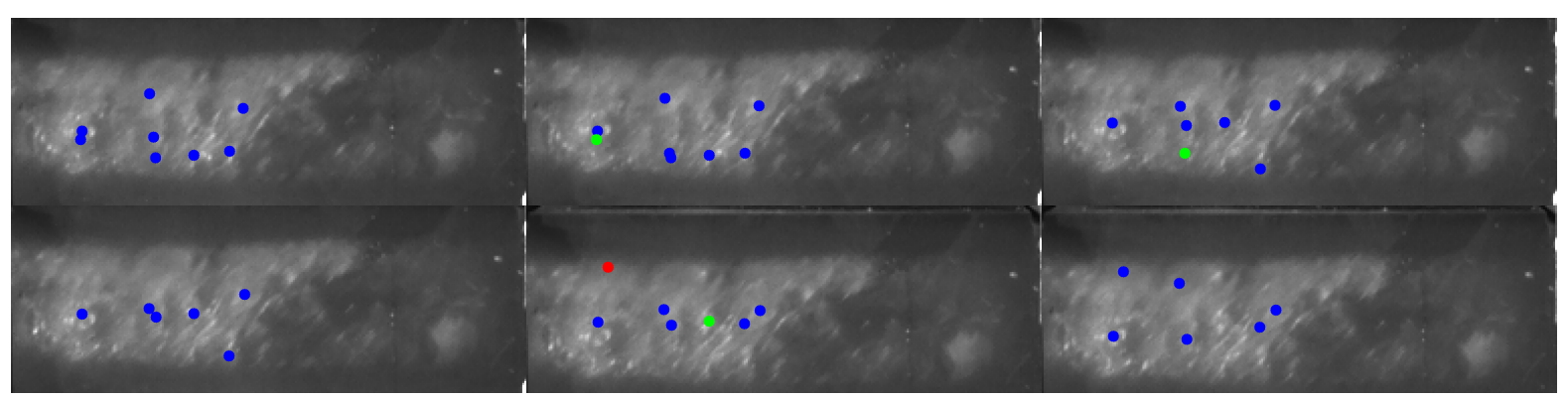

Figure 3: Dense sampled points are tracked using the dense sampling method in (Wang et al., 2013). In this sequence of 6 frames (from left to right over two lines), if a new point is detected, it is colored in red; if the point is tracked in the next frame and the trajectory has not ended yet, the tracked point is in blue otherwise the last point of the trajectory is colored in green.

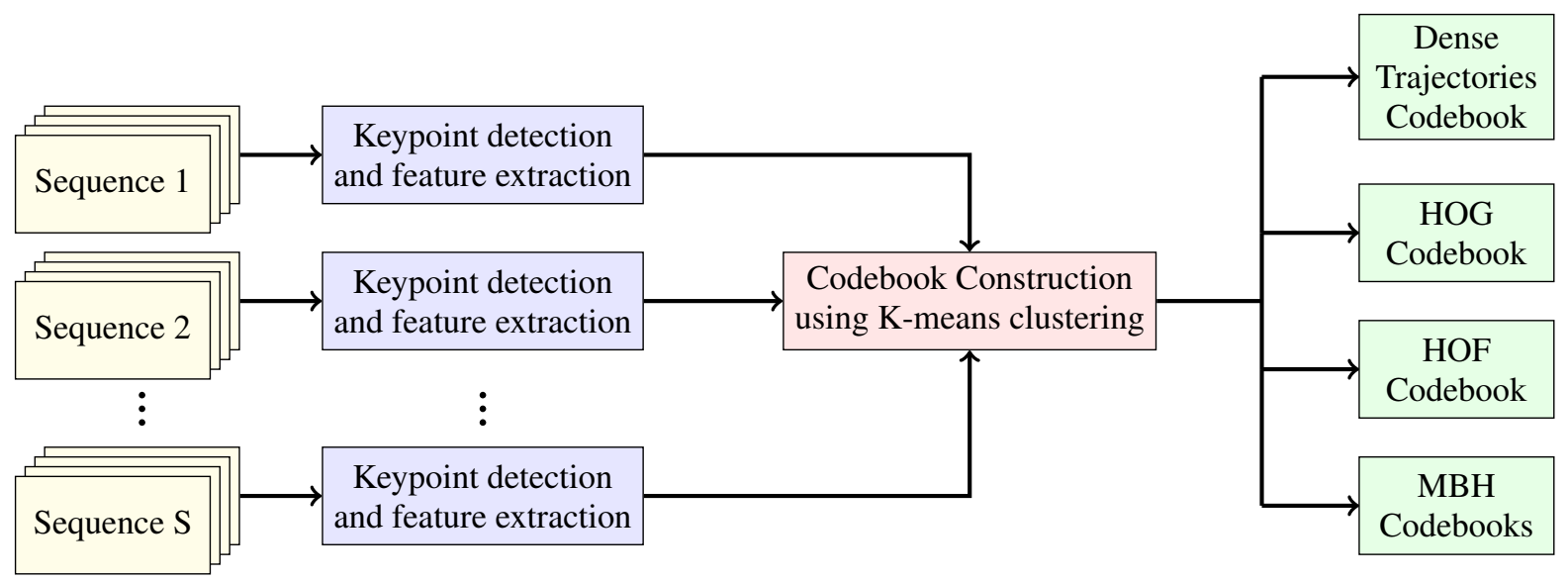

Figure 4: The construction of codebooks based on the calculated trjaectory feature vectors from video sequences.

the temporal and spatial size of the kernel respectively and $N_{i-1}$ is the input dimension (dimension of data or of previous layer, other than spatial and temporal, i.e. number of channels in input images or frames). It is decomposed into $M_{i}$ spatial filters of size $N_{i-1} \times 1 \times$ $d \times d$ and $N_{i}$ temporal filters of size $M_{i-1} \times t \times 1 \times$ 1. The parameter $M_{i}$ is defined as the dimension of a subspace in which the signal is projected between spatial and temporal convolutions and is calculated as: $M_{i}=\frac{t d^{2} N_{i} N_{i-1}}{d^{2} N_{i-1}+t N_{i}}$. A convolution of $(2+1) \mathrm{D}$ is similar to the P3D-A model from (Qiu et al., 2017) but without the bottleneck at the end of a residual layer. Figure 6 illustrates the overall architecture of the $\mathrm{R}(2+1) \mathrm{D}$ model.

\subsubsection{Difference of Gaussian Combined with $\mathbf{R}(\mathbf{2 + 1}) \mathbf{D}$}

In our study, we also propose to pre-process videos before feeding the R(2+1)D network. Indeed, in our opinion, dynamic textures in our dataset are very difficult and the process should focus on important interest points, as for dense trajectory method. The multiscale Laplacian-of-Gaussian (LoG) pyramid is at the basis of many successful keypoint extraction methods, such as SIFT (Lowe, 2004). In our context, we draw inspiration from it in order to select visually interesting parts of the liquid movement. Salient liquid particles appear as specularities, making bright blobs surrounded by darker pixels, which can be detected using the LoG function. We made the hypothesis that using these differences of Gaussian on multiple scales would help the deep learning model.

As in (Lowe, 2004), the LoG is approximated by the Difference of Gaussian for each pair of successive scales as:

$$
D o G(x, y)_{i}=L\left(x, y ; \sigma_{i}\right)-L\left(x, y ; \sigma_{i-1}\right)
$$

where $L\left(\cdot ; \sigma_{i}\right)$ is the convolution of input image with a 2D Gaussian of standard-deviation $\sigma_{i}$. Scales follow a geometric sequence, $\sigma_{i}=\eta \sigma_{i-1}$, with initial scale $\sigma_{1}$ and scale factor $\eta$.

Figure 7 shows the successively smoothed images $L\left(\cdot, \sigma_{i}\right)$ (left column) and corresponding $\operatorname{Do} G(\cdot)_{i}$ (right column). In the DoG images, interesting specularities of liquid particles have negative values, appearing as dark pixels. We believe that the spatiotemporal textures, created by the motion of these specularities, are the main visual cues to extract, in or- 


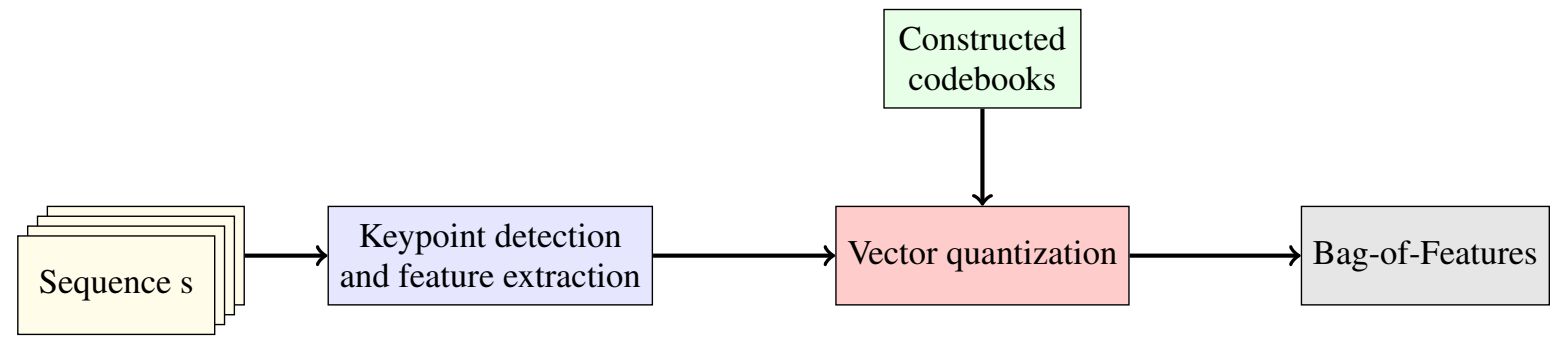

Figure 5: Bag-of-features are created by encoding the trajectory feature vectors inside a sequence thanks to existing codebooks.

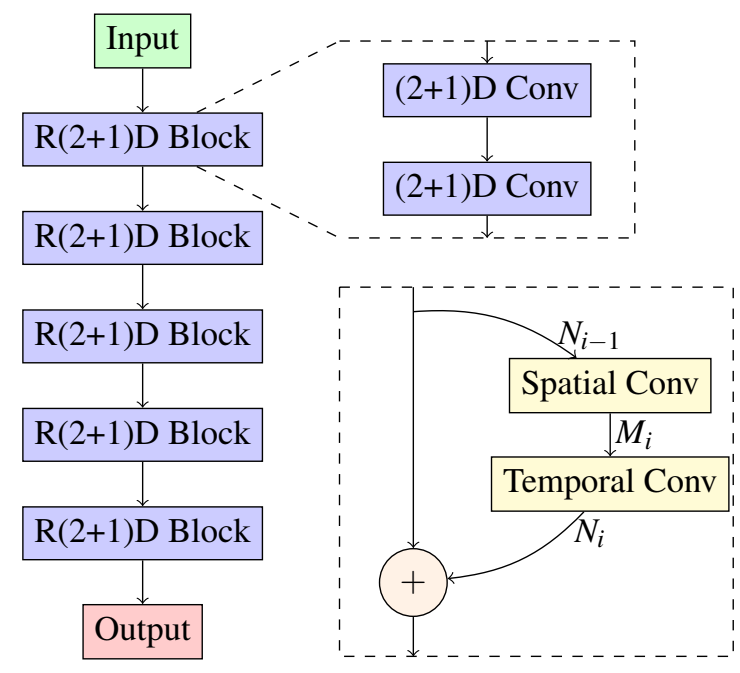

Figure 6: General architecture of R(2+1)D Convolution and the decomposition of a $3 \mathrm{D}$ filters block.

der to classify the motion speed. Hence, we integrate this prior into our deep network: instead of taking the initial grayscale frames as inputs, we feed the network with a thresholded negative multiscale DoG pyramid. Every pixel $(x, y)$ for which $-\operatorname{Do} G(x, y)_{i}<0$ is set to 0 (Figure 8 ). The number of scale factors $F$ is considered as the number of channels, so that the input of the network is a $L \times F \times H \times W$ tensor, where $L$ is the number of frames in a sequence; $F$ is the number of scales; $H$ and $W$ are the height and width of the original frame.

\section{EXPERIMENTS}

In this section, we present an evaluation of the two different methods. The accuracy corresponds to the average of the classification rates for each class (one should remind that a given class corresponds to a fixed motor speed).

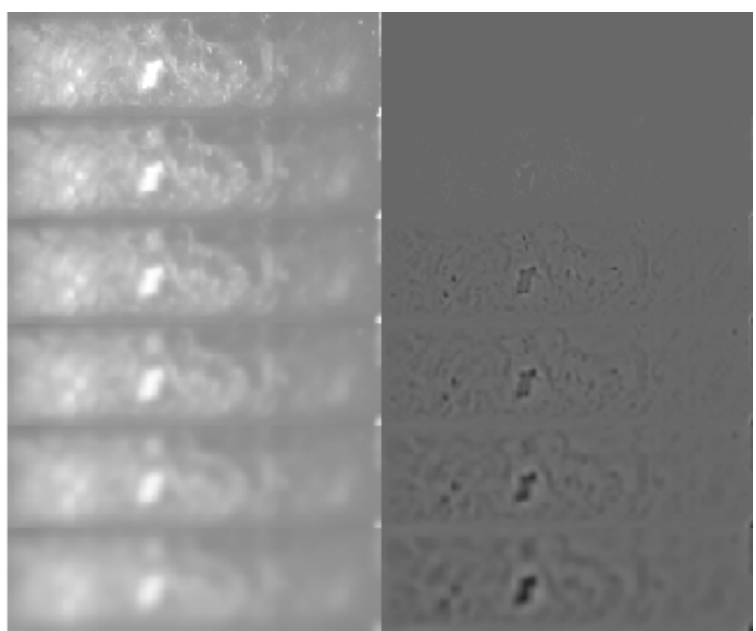

Figure 7: DoG images of a frame at motor speed of 600 rpm with $F=5$ different scales: $\{1, \sqrt{2}, 2,2 \sqrt{2}, 4\}$. First row contains only original frame. In the second row, the first column contains blurred images of the original frame on different scales; the second column contains the DoG for each pair of successive scales.

\subsection{Experimental Setup}

Dense Trajectories. In this method, we keep the default parameters from (Wang et al., 2013). We use 3 spatial scales $\{1, \sqrt{2}, \sqrt{3}\}$, creating 3 spatial levels for each frame. The size of the median filter is fixed to $3 \times 3$. Furthermore, the length of trajectories $L$ (inside sample sequences of 30 frames) is fixed to 15 frames. The cube volume in which a trajectory is extracted is $L \times N \times N$ with $L=15$ and $N=32$. The volume is subdivided into a spatio-temporal grid of size $n_{\tau} \times n_{\sigma} \times n_{\sigma}$ with $n_{\tau}=3, n_{\sigma}=2$. The number of visual word per feature type is analyzed to find out the optimal $M^{\text {opt }}$ for each feature type or their combination. In our experiment, we study $M \in\{1000,1500, \ldots, 6500,7000\}$.

R(2+1)D Network. The R(2+1)D convolutional network takes sequences of 30 frames with a size of $96 \times 256$ as input. In this section, we report the 

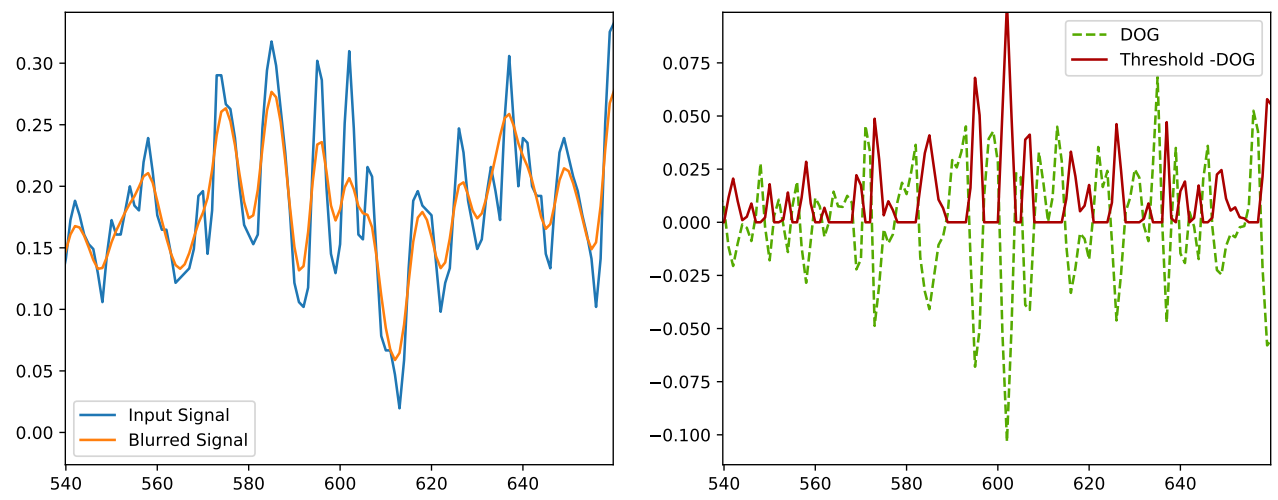

Figure 8: A toy example of the DoG on a 1D signal as pre-processing method before the neural network model.

Table 1: Performance comparison of the different approaches studied on our dataset of liquid-flow videos. The model $R(2+1) D$ with DoG pre-processing outperforms other techniques. The last two columns indicate the accuracy of each method and its average execution time for each clip of 30 frames in the test set. '-': Not available.

\begin{tabular}{c|c|c|c} 
& Methods & Accuracy (\%) & Avg. exe. time per clip (ms) \\
\hline \hline \multirow{3}{*}{ Dense Trajectories } & Displacements & 27.74 & - \\
\cline { 2 - 4 } & HOG & 54.12 & - \\
\cline { 2 - 4 } & HOF & 24.73 & - \\
\cline { 2 - 4 } & MBH & 50.00 & - \\
\cline { 2 - 4 } & Combined features & 59.06 & - \\
\hline \hline \multirow{2}{*}{$\mathrm{R}(2+1) \mathrm{D}$} & Grayscale Frames & 78.06 & 9 \\
\cline { 2 - 4 } & DoG Frames(5 scales) & $\mathbf{8 1 . 3 9}$ & 11
\end{tabular}

results with two kinds of inputs: original sequences of grayscale frames and sequences of DoG frames extracted from original data as explained in section 4.2.2. The sequences of DoG images have 5 channels, corresponding to the 5 scales $\{1, \sqrt{2}, 2,2 \sqrt{2}, 4\}$.

Training and Evaluation. During the training process, other parameters that have not been already fixed are varied and we validate the performance on the validation set to keep the best ones. We then use these parameters to compute the final results on the test set. For the neural network approach, the training is fixed to 50 epochs. The batch size in the training process is 4 , and the ADAM algorithm is used as the optimizer at a fixed learning rate of $10^{-3}$. For the dense trajectories method, the codebook of each descriptor is constructed with $M$ visual words. As for the SVM model, we analyze the performance of the model by varying the penalty parameter $C$ (from 5.0 to 50.0 with a step of 5.0) and the kernel coefficient $\gamma$ (from 0.01 to 0.5 with a step of 0.01 ). The parameters which give the best performance on the validation set are selected for the test set. The best results on each feature type or their combination are shown on Table 1.
All the scripts of our experiments are written in Python. We use Pytorch as a framework and the training of the neural network is done on different types of GPU. For the training (R(2+1)D+DOG), a NVIDIA Tesla K80 is used and NVIDIA Tesla P100 for the training of only grayscale frames. For the testing phase, we only use the Tesla K80 for the evaluation of the trained models. As for the dense trajectories method, the an Intel i7 is used for the whole process.

\subsection{Experimental Results}

The aim of our experiments is to evaluate the correlation between motor speeds and fluids dynamic textures in the cooling gallery in terms of video classification, as a first step before flow patterns and trajectory analysis. For the dense trajectory method, we compare the accuracy by using each individual feature and their combination.

Table 1 illustrates the results obtained by all the methods. As expected, deep learning based methods outperform the hand-crafted features method by at least $20 \%$. Moreover, when comparing the two $\mathrm{R}(2+1) \mathrm{D}$ based methods, the network with the preprocessed inputs with DoG gives a better result by 


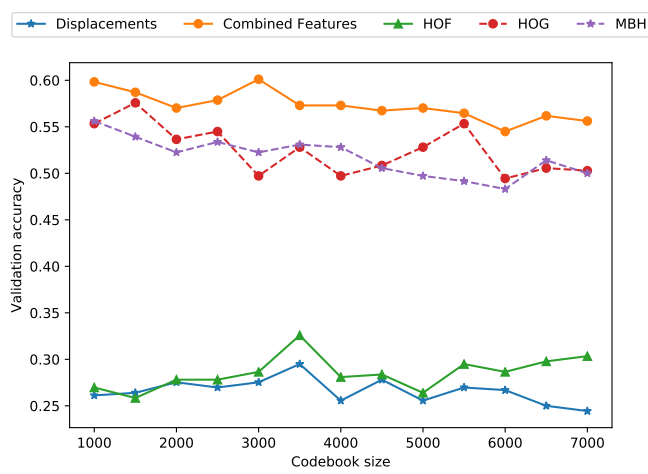

Figure 9: An analysis of the impact of codebook sizes on the accuracy on the validation set.

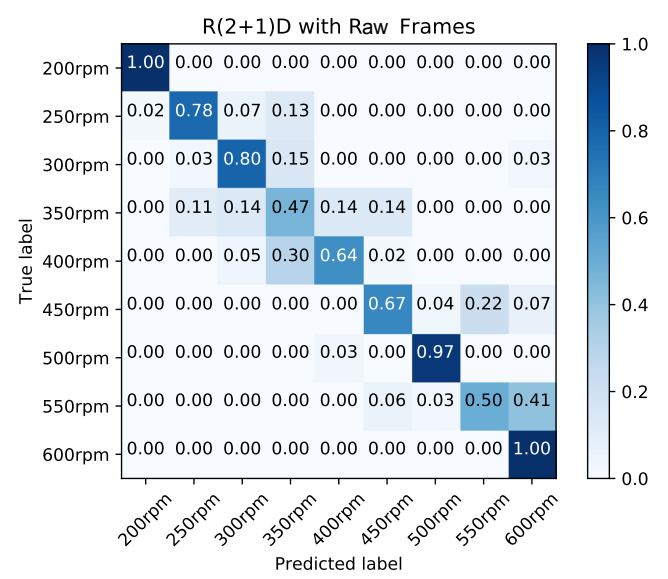

Figure 10: Confusion matrix of $\mathrm{R}(2+1) \mathrm{D}$ on raw grayscale frames.

almost 3\%. This shows the interest of the preprocessing step for such kind of complex spatiotemporal texture analysis. Nevertheless, one should note that such kind of pre-processing to focus on specularities is not compatible with dense trajectories extraction. Indeed, performing such experiments, the accuracy is lower than the one of classical trajectory model, more probably because the number of keypoints extracted from this specularity representation is too small to extract relevant trajectories (this is what we observed).

An analysis of the impact of codebook size is shown in Figure 9. The validation set is used in order to find out the optimal codebook size for each type of feature vector. As shown in this figure, displacement and HOF features show their weakness in classifying the rotation velocity videos. The highest obtained accuracy is found at the codebook size of 3500 (which are always below 35\%). However, when looking at the other feature types (HOG, MBH and combination of these features), it can be recognized that their ac-

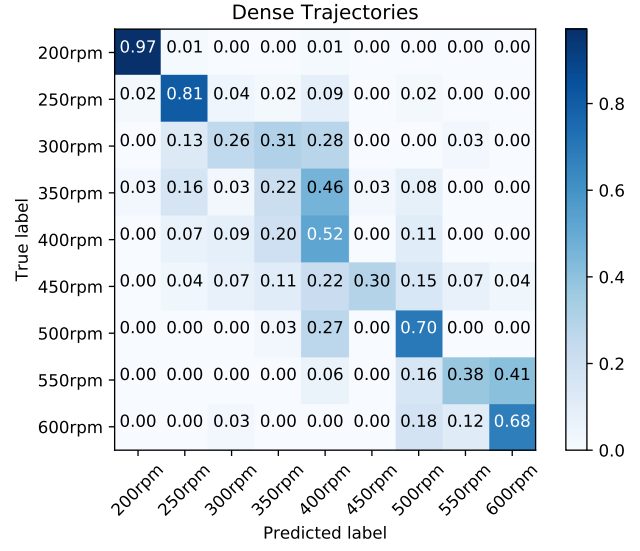

Figure 11: Confusion matrix of dense trajectories method.

curacy on the validation set is much higher than the other two features. Individually, the combination of features always shows its superiority and its highest accuracy at the codebook size of 1000 and 3000 (around $60 \%$ of accuracy). The HOG feature reaches its peak using 1500 visual words $(57.58 \%)$ and the MBH feature has $55.62 \%$ of accuracy at 1000 visual words on the validation set.

Figure 10 shows the normalized confusion matrix of $\mathrm{R}(2+1) \mathrm{D}$ convolution network using only raw grayscale frames as inputs. We note that the model classifies well the sequences corresponding to motor speeds of 200, 300, 500 and $600 \mathrm{rpm}$ (more than $80 \%$ ). Particularly, the model classifies well $100 \%$ of sequences with the motor speeds of 200 and $600 \mathrm{rpm}$ and $90 \%$ for the class of $500 \mathrm{rpm}$. In Figure 12, the confusion matrix of $\mathrm{R}(2+1) \mathrm{D}$ convolution network combined with DoG features is shown. The model classifies perfectly the data from the class $200 \mathrm{rpm}$ $(100 \%)$ and pretty well with some other classes like $250,400,500$ and $600 \mathrm{rpm}$ (around 90\% of accuracy). We can also see that the results of this model are distributed more equally than the model without the pre-processing step (59\%-100\% compared with $47 \%$ $100 \%)$. Considering, the dense trajectory method (Figure 11), it can only classify well the first class $200 \mathrm{rpm}(96 \%)$ and somehow not bad the classes of $250 \mathrm{rpm}$ and $600 \mathrm{rpm}$ (72\% and $71 \%$ respectively).

From these experimental results, the deep learning-based methods again show theirs efficiency on dynamic texture classification task over handcrafted features. This may be due to two reasons. Firstly, contrarily to our hypotheses, dense trajectories are probably not the right way to describe dynamic textures for such kind of liquid movements. Secondly, the extraction of such trajectories is possibly not good enough with state-of-the-art approach 


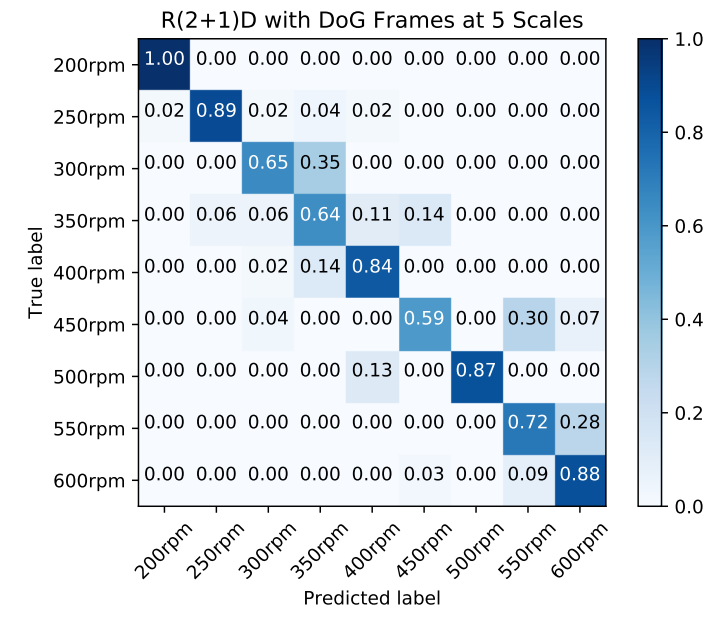

Figure 12: Confusion matrix of $\mathrm{R}(2+1) \mathrm{D}$ on DoG frames.

and then it should be adapted. It could be for example because of the fixed trajectory length. Indeed, we may lose some trajectories that have length shorter or longer than 15 frames and therefore a lot of interesting spatio-temporal information may be lost during the tracking process. This might be particularly true in our case when the two phases of the mechanical process are creating droplets and bubbles.

\section{CONCLUSIONS}

In this paper, a new dataset based on two-phased flow visualization inside a simulation of a cooling gallery is proposed to the community. This dataset opens a field for research on dynamic texture analysis and especially for flow patterns extraction and analysis of their trajectories. Here, flow patterns are used for a classification task: two classical computer vision techniques are studied in order to validate the fact that there exists a correlation between the motor speed and the movements of fluids in a two-phase flow engineering process. The first approach, based on the state-ofthe-art approach to extract and characterize trajectories, seems not to work well on fluids particles even if there exists room for improvements. This represents a first challenging task since being able to extract and analyze these trajectories is really important for the considered application domain: being able to analyze and describe the behaviour of particules and thus their trajectories could be useful for engineers. On the other side, deep learning approaches based on $\mathrm{R}(2+1) \mathrm{D}$ convolutions give better results even if not completely satisfying. Consequently, we propose in this study to improve the approach adding a prepro- cessing step that changes the original videos representation to highlight specularities of fluids, thanks to DoG. The counterpart of this deep method is the loss of explanability of the decision and modeling process. Then, another challenging task brought by this study is how to use deep features to extract flow patterns and their trajectories to make further analysis possible.

\section{ACKNOWLEDGEMENTS}

This work was supported by University of Zhejiang and Haoyi Niu in particular. We gratefully acknowledged the support of his work with the video data used for this research.

\section{REFERENCES}

Andrearczyk, V. and Whelan, P. F. (2018). Convolutional neural network on three orthogonal planes for dynamic texture classification. Pattern Recognition, 76:36-49.

Crivelli, T., Cernuschi-Frias, B., Bouthemy, P., and Yao, J.-F. (2013). Motion Textures: Modeling, Classification, and Segmentation Using Mixed-State Markov Random Fields. SIAM Journal on Imaging Sciences, 6(4):2484-2520.

Dalal, N. and Triggs, B. (2005). Histograms of Oriented Gradients for Human Detection. In 2005 IEEE Computer Society Conference on Computer Vision and Pattern Recognition (CVPR'05), volume 1, pages 886-893. IEEE.

Dalal, N., Triggs, B., Schmid, C., Dalal, N., Triggs, B., Schmid, C., Detection, H., and Oriented, U. (2006). Human Detection Using Oriented Histograms of Flow and Appearance. European Conference on Computer Vision (ECCV), 1(1):428-441.

Farnebäck, G. and Farneb, G. (2003). Two-Frame Motion Estimation Based on. Lecture Notes in Computer Science, 2003(1):363-370.

Grabner, H., Grabner, M., and Bischof, H. (2006). RealTime Tracking via On-line Boosting. In Procedings of the British Machine Vision Conference 2006, pages 6.1-6.10. British Machine Vision Association.

Jansson, Y. and Lindeberg, T. (2018). Dynamic Texture Recognition Using Time-Causal and Time-Recursive Spatio-Temporal Receptive Fields. Journal of Mathematical Imaging and Vision, 60(9):1369-1398.

Jianbo Shi and Tomasi (1994). Good features to track. In Proceedings of IEEE Conference on Computer Vision and Pattern Recognition CVPR-94, volume 169, pages 593-600. IEEE Comput. Soc. Press.

Krizhevsky, A., Sutskever, I., and Hinton, G. E. (2017). ImageNet classification with deep convolutional neural networks. Communications of the ACM, 60(6):84-90. 
Laptev, I., Marszalek, M., Schmid, C., and Rozenfeld, B. (2008). Learning realistic human actions from movies. In 2008 IEEE Conference on Computer Vision and Pattern Recognition, pages 1-8. IEEE.

Lowe, D. G. (2004). Distinctive image features from scaleinvariant keypoints. International Journal of Computer Vision, 60(2):91-110.

Lu, Z., Xie, W., Pei, J., and Huang, J. J. (2007). Dynamic texture recognition by spatio-temporal multiresolution Histograms. Proceedings - IEEE Workshop on Motion and Video Computing, MOTION 2005, (200338):241246.

Nelson, R. C. and Polana, R. (1992). Qualitative recognition of motion using temporal texture. CVGIP: Image Understanding, 56(1):78-89.

Qi, X., Li, C. G., Zhao, G., Hong, X., and Pietikäinen, M. (2016). Dynamic texture and scene classification by transferring deep image features. Neurocomputing.

Qiu, Z., Yao, T., and Mei, T. (2017). Learning SpatioTemporal Representation with Pseudo-3D Residual Networks. Proceedings of the IEEE International Conference on Computer Vision, 2017-Octob:55345542.

Renaud, P. and Chetverikov, D. (2005). Using Normal Flow and Texture Regularity. Pattern Recognition and Image Analysis, (June 2005):223-230.

Tran, D., Bourdev, L., Fergus, R., Torresani, L., and Paluri, M. (2015). Learning spatiotemporal features with 3D convolutional networks. Technical report, Santiago.

Tran, D., Wang, H., Torresani, L., Ray, J., LeCun, Y., and Paluri, M. (2018). A Closer Look at Spatiotemporal Convolutions for Action Recognition. In 2018 IEEE/CVF Conference on Computer Vision and Pattern Recognition, pages 6450-6459. IEEE.

Wang, H., Kläser, A., Schmid, C., and Liu, C.-L. (2013). Dense Trajectories and Motion Boundary Descriptors for Action Recognition. International Journal of Computer Vision.

Zhang, J., Marszałek, M., Lazebnik, S., and Schmid, C. (2007). Local features and kernels for classification of texture and object categories: A comprehensive study. International Journal of Computer Vision, 73(2):213238. 